\title{
TABLES DE PRODUCTION PROVISOIRES POUR L'ÉPICÉA COMMUN DANS LE NORD-EST DE LA FRANCE
}

\author{
N. DECOURT \\ avec la collaboration technique de F. Michaux et A. Tisserand \\ Station de Sylviculture et de Production, \\ Centre national de Recherches forestières, I.N.R.A., \\ 54 - Champenoux, par Einville
}

\begin{abstract}
RÉSUMÉ
Ces tables de production provisoires concernent des peuplements purs et équiennes. Elles ont été entièrement calculées à partir de placettes temporaires.

La méthode est fondée sur le calcul de relations, par la méthode de régression multiple, entre les variables suivantes : âge, hauteur moyenne, hauteur dominante, nombre de tiges à l'hectare, circonférence moyenne et volume.

Ces équations permettent le calcul de relations fondamentales exprimant la croissance en hauteur, la circonférence moyenne et le volume.

A partir de ces relations et après le choix d'un régime d'éclaircie, on peut calculer les tables usuelles.
\end{abstract}

La construction de tables de production pour les principales essences forestières françaises, se poursuit avec cette table, valable pour l'Épicéa Commun (Picea abies, L.) dans le nord-est de la France. La méthode employée a déjà été utilisée pour d'autres tables de production françaises : Pin Sylvestre et Pin Laricio en Sologne, Douglas dans le nord-est du Massif Central, Pin maritime dans le sud-ouest de la France. On se reportera à ces précédentes études pour tout ce qui concerne l'implantation et la mesure des placettes et pour le détail des procédés de calcul. (Decourt N., 1965, 1967; Decourt N. et Lemoine B., 1969). De plus, on trouvera un exposé critique de la méthode dans ce même numéro des Annales. 


\section{I. - RÉCOLTE DES DONNÉES \\ 1.1. - Limites de la région concernée}

Les placettes ont été installées dans les départements suivants : Ardennes, Côte d'Or, Haute-Marne, Meurthe-et-Moselle, Meuse, Moselle, Bas-Rhin, Vosges. Les stations sont variées depuis les plateaux calcaires de l'est jusqu'aux Vosges gréseuses et cristallines, en passant par la plaine Lorraine et depuis les Ardennes jusqu'au nord de la Bourgogne.

Les peuplements d'altitude supérieure à 900 mètres ont été éliminés. Aucun peuplement ne provient non plus de la plaine d'Alsace.

127 placettes semi-permanentes ont été mesurées, comme dans le cadre des précédentes études, dont 28 sur les plateaux calcaires, au cours d'un travail sur la productivité de cette zone (Decourt N. et Le Tacon F., 1970).

\section{2. - Placettes semi-permanentes et microplacettes}

Le dispositif de placettes semi-permanentes a été complété, pour la zone calcaire, par les données provenant de 52 «microplacettes » supplémentaires, mais seulement pour préciser la liaison : âge, hauteur dominante, indice de productivité. Ces données supplémentaires proviennent également du travail cité ci-dessus. Rappelons que ces «microplacettes» sont constituées par les 50 arbres les plus proches d'une fosse pédologique destinée à l'étude du sol, ce qui représente des surfaces de 3 à 5 ares. Elles servent surtout à déterminer la hauteur dominante du peuplement et ne permettent pas d'évaluer un volume à l'hectare, comme les placettes semi-permanentes, maintenant traditionnelles. Dans les conditions d'homogénéité remarquables des plantations d'Épicéas du nord-est, on a pu montrer que l'évaluation de la hauteur dominante était parfaitement comparable dans les microplacettes et dans les placettes semi-permanentes. On s'est donc servi de ces données supplémentaires.

\section{3. - Analyses de tiges}

Les résultats de 42 analyses de tiges d'arbres dominants provenant des placettes semipermanentes ont été utilisés également, pour mieux préciser la forme de la courbe de croissance en hauteur.

La répartition des placettes semi-permanentes, par département, figure dans le tableau 1 . Pour la répartition des microplacettes, on se reportera à l'étude déjà citée.

\section{II. - MÉTHODE UTILISÉE ET CONSTRUCTION DE LA TABLE}

\section{1. - Principes généraux}

Pour la définition précise des variables dendrométriques, on se reportera à l'annexe 1 où on trouvera également le rappel des symboles utilisés pour les désigner. 
TABLEAU 1

Répartition des placettes semi-permanentes par Département Distribution of the temporary plots by departments

\begin{tabular}{l|r|r}
\hline \multicolumn{1}{c|}{ Département } & Total & Plateaux calcaires \\
\cline { 2 - 3 } & & \\
\cline { 2 - 2 } Ardennes (08) & 9 & - \\
Côte d'Or (21) & 5 & 5 \\
Haute-Marne (52) & 6 & 6 \\
Meurthe-et-Moselle (54) & 19 & 3 \\
Meuse (55) & 13 & 13 \\
Moselle (57) & 17 & - \\
Bas-Rhin (67) & 48 & 1 \\
Vosges (88) & 127 & 28 \\
& & \\
& & \\
\end{tabular}

La méthode s'appuie, rappelons-le, sur l'établissement de trois ensembles de relations décrivant les liaisons entre les valeurs des variables.

- Un ensemble [S] de relations indépendantes de l'âge et de la station.

- Un ensemble [M], limité ici à une seule relation entre la hauteur dominante, l'âge et un indice de productivité I, caractérisant la station.

- Un ensemble [E], décrivant la Sylviculture proposée dans la table.

Ces relations, présentées sous forme d'équations ou de tableaux sont rassemblées dans le Tableau 2. On trouvera, ci-après, quelques précisions sur leur établissement.

TABLEAU 2

Relations utilisées pour la construction de la table de production pour l'Épicéa Commun dans le Nord-Est de la France. (127 placettes)

The different relationships idilized for the computation of a yield-table for Norway spruce in the northeast of France (127 plots).

[S]
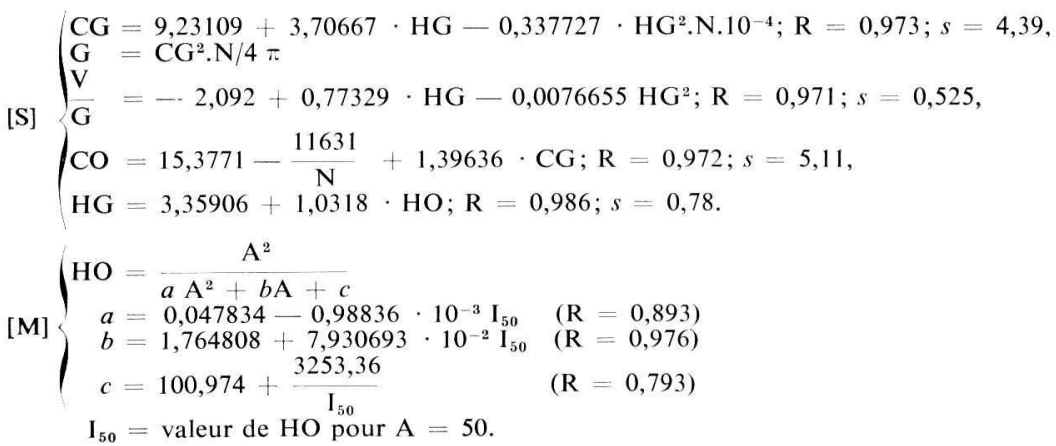

\begin{tabular}{c|c|c|r|r}
\hline Classe & $I_{50}$ & a & b & \\
\hline & & & & \\
1 & 30 & 0,018035 & 0,626314 & 21,574223 \\
2 & 27 & 0,021596 & 0,340599 & 37,782744 \\
3 & 24 & 0,024661 & 0,094661 & 56,908349 \\
4 & 21 & 0,027468 & $-0,130592$ & 80,796741 \\
5 & 18 & 0,030145 & $-0,345361$ & 112,552625 \\
6 & 15 & 0,032776 & $-0,556463$ & \\
\hline \hline
\end{tabular}




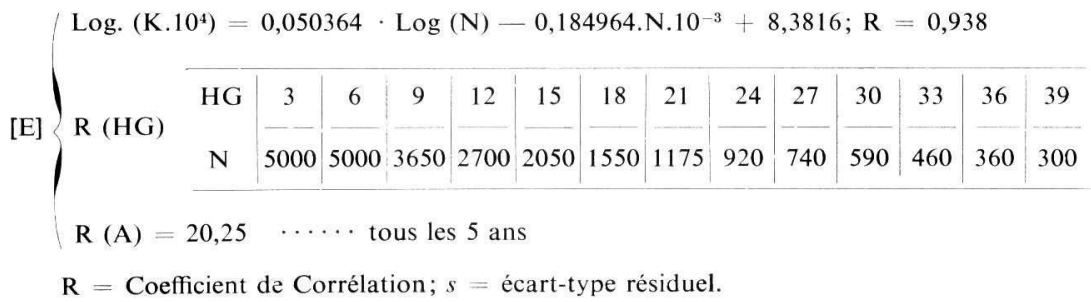

\section{2. - Relation décrivant les peuplements quel que soit leur âge et leur productivité : [S]}

\subsection{Calcul des équations.}

Ces relations ont été obtenues, comme pour les tables précédemment publiées, par la méthode d'ajustement dite de régression progressive ${ }^{1}$ (Programme REPRG de la Station de Biométrie du C.N.R.F.). Après plusieurs essais, on a choisi les relations à la fois les plus simples et les meilleures, le tri s'effectuant sur la valeur du coefficient de corrélation et sur celle de l'écart-type résiduel.

Comme pour les autres essences, on a constaté :

- que les ajustements sont excellents $(\mathrm{R}>0,97)$;

- que 1'âge n'intervient pas de façon significative dans ces équations, dès Iors qu'y figure déjà la hauteur moyenne $\mathrm{HG}$ ou la hauteur dominante $\mathrm{HO}$.

\subsection{Comparaison entre la zone des plateaux calcaires et la zone non calcaire.}

Un problème se posait : fallait-il construire une table de production pour la zone des plateaux calcaires et une autre, différente, pour les autres régions, notamment pour la région vosgienne?

Pour répondre à cette question, on a employé une méthode classique de comparaison des équations de régression (KozAK A., 1970), qu'on avait déjà, du reste, utilisée pour comparer les Pins maritimes des «Landes » à ceux des « Dunes ». Il est apparu qu'aucune différence significative n'existait entre les peuplements d'Épicéa des plateaux calcaires et les autres.

Par contre, les quelques peuplements d'altitude dont nous disposions dans les hautesVosges, se sont révélés différents des autres, ce qui nous a conduit à limiter la zone de validité de notre table aux altitudes inférieures à 900 mètres.

Ajoutons que la relation retenue pour le cubage des peuplements :

$$
\frac{\mathrm{V}}{\mathrm{G}}=-2,092+0,77329 \cdot \mathrm{HG}-0,0076655 \cdot \mathrm{HG}^{2}
$$

1. Les données utilisées peuvent être fournies sur demande. 
est sans doute valable dans presque toute la France, comme l'a montré le même type de comparaison effectuée sur 234 placettes, regroupées en 5 régions, dont notamment les deux zones qui nous intéressent ici (DeCOuRT N., 1971).

\section{3. - Relation $H O=f(\mathrm{~A}, \mathrm{I})$}

On a utilisé les 80 couples (HO, A) tirés des placettes (semi-permanentes et microplacettes) sur plateau calcaire et les 99 couples tirés des autres placettes semi-permanentes, ainsi que 42 analyses de tiges regroupées en trois séries d'indice de productivité comparable ( 2 pour les plateaux calcaires, 1 pour les Vosges).

Après avoir reporté les points représentatifs des 80 et 99 couples, on a tracé les courbes limitant chaque nuage de points vers le haut et vers le bas. On disposait ainsi de 4 courbes de croissance provenant des nuages de points et de 3 courbes provenant de moyennes d'analyses de tiges, dont au total 4 courbes propres aux plateaux calcaires et 3 pour le reste de la région.

Une équation de la forme

$$
\mathrm{HO}=\frac{\mathrm{A}^{2}}{\mathrm{aA}^{2}+\mathrm{bA}+\mathrm{c}}
$$

a été calculée pour chacune de ces courbes, en déterminant les constantes a, b, c imposant à ces courbes de passer par trois points d'abcisses $\mathrm{A}=30, \mathrm{~A}=40, \mathrm{~A}=50$.

L'étude des 7 triplets $(a, b, c)$ caractérisant chacune des 7 courbes a montré qu'on pouvait exprimer, dans de bonnes conditions, les trois coefficients $a, b$, c en fonction d'un indice de productivité $I_{50}$, choisi comme la valeur de HO à l'âge de 50 ans. (Fig. 1.)

On obtient ainsi :

$$
\begin{aligned}
& \mathrm{a}=0,047834-0,98836 \cdot 10-^{3} \mathrm{I}_{50} \\
& \mathrm{~b}=1,764808+7,930693 \cdot 10-^{2} \mathrm{I}_{50} \\
& \mathrm{c}=100,974+\frac{3253,36}{\mathrm{I}_{50}}
\end{aligned}
$$

L'ensemble de ces trois équations et l'équation (1) déterminent la relation :

$$
\mathrm{HO}=\mathrm{f}\left(\mathrm{A}, \mathrm{I}_{50}\right)
$$

D'autre part, rien n'indique qu'une différence existe entre les courbes des peuplements sur plateaux calcaires et les autres, bien qu'évidemment aucun test objectif n'ait pu être fait par cette méthode. On a donc retenu une seule relation valable pour l'ensemble des données.

Il faut cependant remarquer que les équations (2) ayant elles-mêmes été ajustées par la méthode de régression linéaire, les coefficients $a, b, c$ qu'elles déterminent pour un $\mathrm{I}_{50}$ donné ne correspondent pas exactement au passage de la courbe (1) par le point $(\mathrm{A}=50$, $\mathrm{HO}=\mathrm{I}_{50}$ ). On peut cependant calculer facilement la correction à faire, c'est-à-dire la valeur $I_{50}^{\prime}$ à utiliser dans les équations (2) pour obtenir une courbe (1) passant exactement 


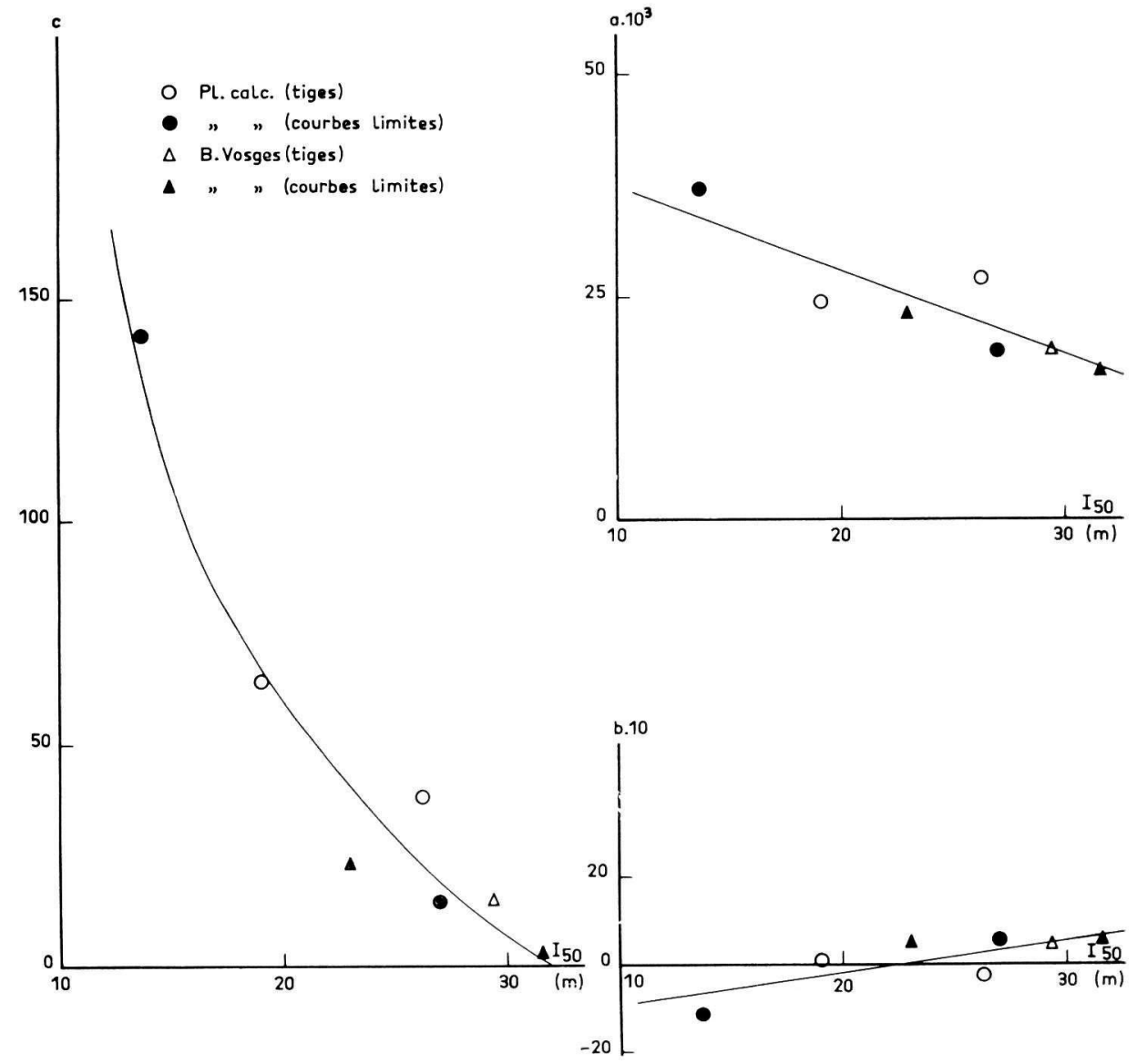

FIG. 1. - Valeurs des coefficients a, b, c en fonction de l'indice de productivité (voir p. 5)

FIG. 1. - Values of the coefficients a, b, c in relation with site index (see p. 5)

par le point choisi $\left(\mathrm{A}=50, \mathrm{HO}=\mathrm{I}_{50}\right)$ pour définir une classe de productivité. C'est ce qu'on a fait pour calculer les 6 équations (1) correspondant aux 6 classes de productivité de la table [cf. Annexe 2 et fig. 2].

\section{4. - Définition des éclaircies [E]}

La table intermédiaire (indépendante de l'âge) a été calculée pour des valeurs de HG espacées de $3 \mathrm{~m}$ en $3 \mathrm{~m}$.

Dans les tables, les éclaircies sont prévues tous les 5 ans. En fait, par construction, ces dernières tables étant tirées de la table intermédiaire, on peut choisir une autre succession de rotations. Pour obtenir des rotations de 10 ans par exemple, on fera la somme des éclaircies de deux lignes successives, le peuplement sur pied après éclaircie n'étant — en théorie pas modifié par cette opération. 
L'intensité de l'éclaircie est modérée. Elle correspond cependant à des interventions sensiblement plus fortes que celles effectivement pratiquées en moyenne. Le choix de ce régime provient de l'étude de la sylviculture pratiquée dans ce qui nous a paru les propriétés les mieux gérées.

Il en va de même du choix de la nature de l'éclaircie, assez nettement par le bas. Le modèle choisi est tiré de la table de production allemande de ZimmerLe (1947) et correspond également à la moyenne des valeurs du rapport $\mathrm{K}=\frac{\mathrm{V}_{\mathrm{m}}}{\mathrm{VG}}$ trouvé dans les placettes où on a pu l'évaluer. Ce type d'éclaircie correspond du reste, en gros, à ce qui se pratique en Europe moyenne, à de petites différences près. En Grande-Bretagne, au contraire, l'éclaircie pratiquée, même dans l'Épicéa commun, est nettement par le haut.

Il n'est pas indifférent de remarquer que cette façon de décrire la nature de l'éclaircie est très efficace. Si on calcule, en effet, les valeurs de $\mathrm{K}$ pour la table de production de ZimMERLE (1947), et qu'on ajuste ensuite, par régression, une équation, telle que celle du tableau 2 exprimant $\mathrm{K}$ en fonction de $\mathrm{N}$ (nombre d'arbres sur pied avant l'éclaircie), on trouve un ajustement excellent $(\mathrm{R}=0,938 ; \mathrm{n}=50$ ), alors que, bien entendu ZimmerLE n'a pas choisi ce critère a priori. Il en va de même pour bon nombre d'autres tables de production.

\section{III. - RÉSULTATS OBTENUS - COMPARAISON AVEC LES TABLES ÉTRANGÈRES LIMITES DE VALIDITÉ}

\section{1. - Les tables}

On trouvera les tables ainsi calculées dans l'Annexe 2. Elles sont réparties en 6 classes de productivité. En fait, les peuplements de la classe $1(\mathrm{ou}>1)$ n'existent pas sur les plateaux calcaires, ou sont très rares. Inversement, la classe 6 (ou $<6$ ) est peu représentée dans la zone vosgienne. Le tableau 3 résume la répartition des placettes par classe de pro-

TABLEAU 3

Répartition des placettes par classes de productivité Distribution of the Plots by yield-classes

\begin{tabular}{|c|c|c|c|c|c|c|c|c|}
\hline \multirow{2}{*}{ Classes } & \multicolumn{2}{|c|}{ Vosges } & \multirow{2}{*}{$\begin{array}{c}\text { Pl. } \\
\text { calcaires } \\
\text { nb }\end{array}$} & \multirow{2}{*}{$\begin{array}{c}\begin{array}{c}\text { micro } \\
\text { placettes }\end{array} \\
\% \\
\%\end{array}$} & \multirow{2}{*}{$\begin{array}{c}\text { Pl. } \\
\text { calcaires } \\
\text { nb }\end{array}$} & \multirow{2}{*}{$\begin{array}{c}\text { Pl. semi } \\
\text { perman. } \\
\frac{\%}{\%}\end{array}$} & \multicolumn{2}{|c|}{ Total } \\
\hline & $\mathrm{nb}$ & $\%$ & & & & & $\mathrm{nb}$ & $\%$ \\
\hline $\begin{array}{l}>I \\
\text { I-II } \\
\text { II-III } \\
\text { III-IV } \\
\text { IV-V } \\
\text { V-VI } \\
<\text { VI }\end{array}$ & $\begin{array}{r}10 \\
36 \\
36 \\
16 \\
1 \\
-\end{array}$ & $\begin{array}{c}10,1 \\
36,4 \\
36,4 \\
16,1 \\
1,0 \\
-\end{array}$ & $\begin{array}{r}-4 \\
19 \\
21 \\
19 \\
8 \\
9\end{array}$ & $\begin{array}{r}- \\
5,0 \\
23,8 \\
26,2 \\
23,8 \\
10,0 \\
11,2\end{array}$ & $\begin{array}{r}-1 \\
11 \\
9 \\
6 \\
1 \\
\end{array}$ & $\begin{array}{r}- \\
3,6 \\
39,3 \\
32,1 \\
21,4 \\
3,6\end{array}$ & $\begin{array}{r}10 \\
40 \\
55 \\
37 \\
20 \\
8 \\
9\end{array}$ & $\begin{array}{r}5,6 \\
22,3 \\
30,7 \\
20,7 \\
11,2 \\
4,5 \\
5,0\end{array}$ \\
\hline Totaux & 99 & 100,0 & 80 & 100,0 & 28 & 100,0 & 179 & 100,0 \\
\hline
\end{tabular}


ductivité. Chaque colonne de table correspond à l'évolution dans le temps d'une variable dendrométrique (voir annexe 1). La colonne ECL. correspond au pourcentage de la production totale enlevée en éclaircie.

\section{2. - Comparaisons avec quelques résultats obtenus à l'étranger}

Il existe de très nombreuses tables de production pour l'Épicéa commun dans les principaux pays d'Europe et il ne saurait être question ici de toutes les comparer à nos résultats. Nous nous sommes donc bornés à effectuer quelques comparaisons avec les tables de ZimmerLe (1947) pour le Württemberg et avec les travaux belges sur l'Épicéa dans les Ardennes : Delvaux J. (1968) et plus récemment Dagnelie P., Nivelle J.-L., Rondeux J. et Thill A. (1970). Les deux régions concernées sont en effet immédiatement voisines du nord-est de la France.

\subsection{Croissance en hauteur.}

Les figures 3A et 3B montrent une assez bonne similitude de l'allure de la croissance en hauteur de l'Épicéa dans les trois régions ${ }^{1}$ avec cependant une certaine tendance à une baisse de croissance plus précoce pour les tables françaises.

La classe de productivité no 3 de la table française correspond sensiblement aux résultats des auteurs belges. D'une façon générale, le niveau de productivité est plus élevé en France que dans le Württemberg (Table de ZimmerLe).

Compte tenu de la marge d'incertitude qui règne dans l'établissement de ces courbes, on peut estimer que la concordance est satisfaisante entre les résultats obtenus dans ces trois régions voisines.

\subsection{Croissance en volume.}

La figure 4 permet de comparer les courbes exprimant la production totale en volume en fonction de la hauteur moyenne.

On note une bonne concordance entre la table allemande, la table française et le résultat d'une évaluation directe faite à partir des placettes les plus denses du nord-est de la France dans une étude précédente (Decourt N., Le Tacon F., 1970). Par contre, la courbe belge est sensiblement au-dessous des précédentes. Delvaux J. estime, cependant, que cette différence tient à la nature de son échantillon et il précise même « que les tables de ZimmerLE sont celles avec lesquelles les productions observées en Ardennes concordent le mieux » (page 54).

De la combinaison des deux séries de comparaisons ci-dessus, il résulte que la principale différence de nos tables avec celles de nos voisins serait une chute de la production courante annuelle plus précoce en France qu'en Württemberg notamment. Après les secondes séries de mesures dans nos placettes, ce point pourra être précisé dans de meilleures conditions.

1. Delvaux J. ne donne pas de résultats pour la croissance en hauteur. 


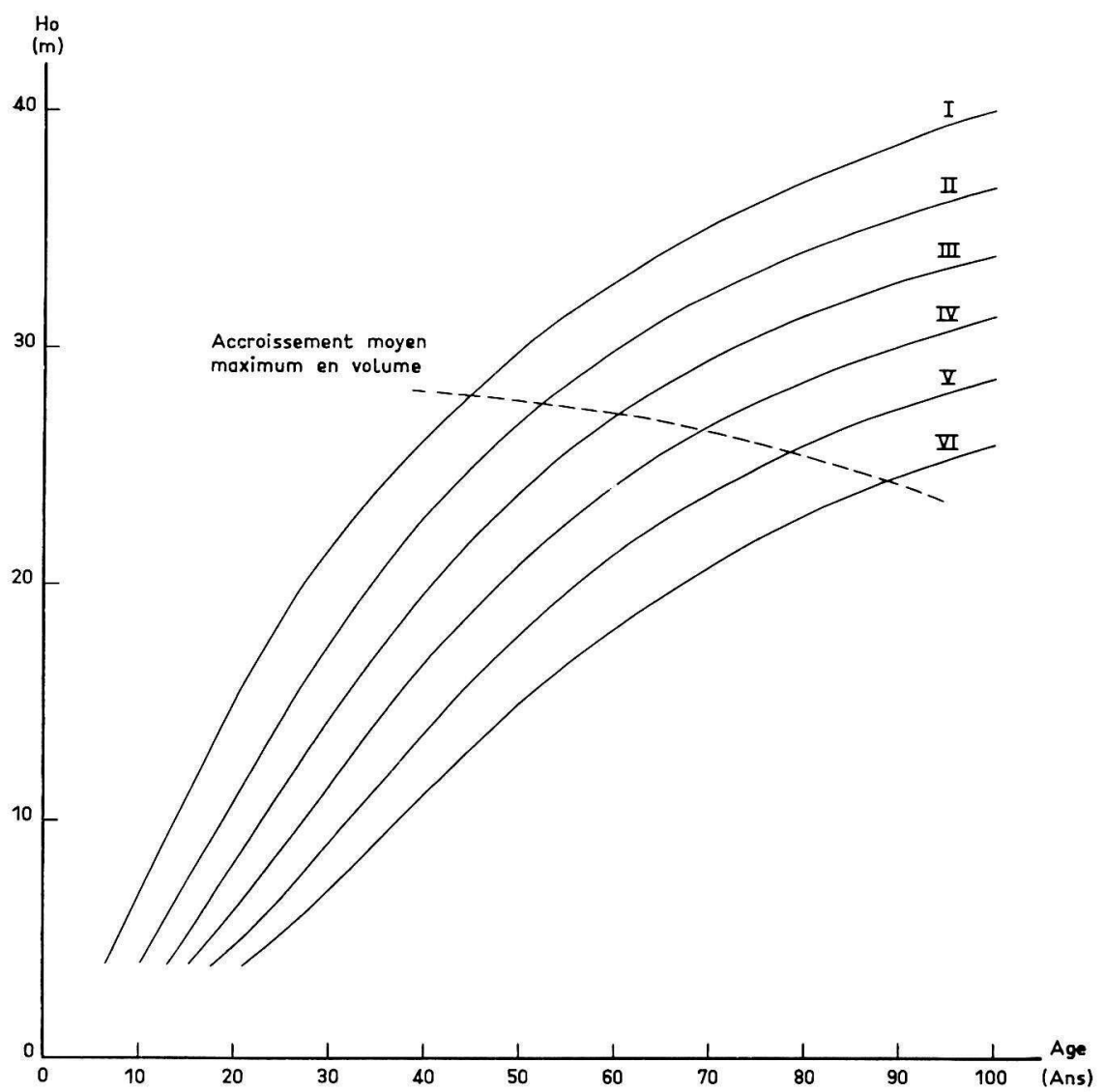

FIG. 2. - Courbes de croissance en hauteur et classes de productivité

FIG. 2. - Yield-class curves 

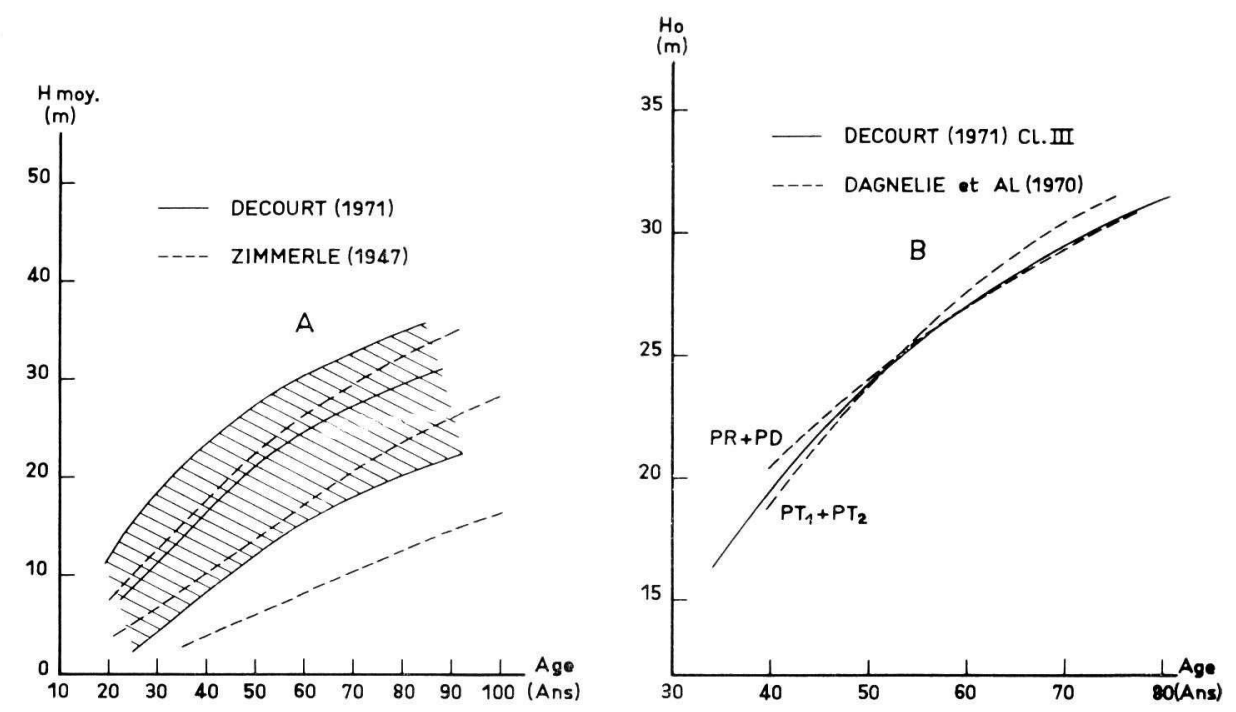

FIG. 3A. - Courbe de croissance en hauteur dans le Württemberg (Classe I, 3, 5) et dans le N.-E. de la France (Classe 1, 3, 6)

FIG. 3B. - Courbes de croissance en hauteur dans les Ardennes Belges (types PR + PD sur sols bruns acides et types $\mathrm{PT}_{1}+\mathrm{PT}_{2}$ sur sols hydromorphes) et dans le N.-E. de la France (Classe 3)

FIG. 3 A. - Height/Age curves in Württemberg (class 1,3,5) und in Northeast of France (class 1, 3, 6) FIG. 3 B. - Height/Age curves in Belgian Ardennes (PR $+\mathrm{PD}$ on acid brown soils and $\mathrm{PT}_{1}+\mathrm{PT}_{2}$ on hydromorphic soils) and in Northeast of France (class 3).

\section{3. - Limites de validité}

Les tables sont valables pour le nord-est de la France, dans les limites géographiques précisées au début de cet article. Les comparaisons qui viennent d'être faites, montrent, cependant, que les conditions de croissance de l'Épicéa sont relativement homogènes dans une région plus large. Des études ultérieures, notamment dans le Jura (boisements artificiels des plateaux), montreront si on peut étendre ces résultats aux régions voisines. Il sera également intéressant de faire des comparaisons avec la table de production, en préparation, pour le Limousin.

En ce qui concerne l'échantillon dont nous disposions, le tableau 4 précise le domaine de variation dans lequel on peut considérer comme valables nos équations de base. Nous n'avons pas disposé d'un nombre de peuplements suffisants, soumis à des éclaircies fortes. Seule l'expérimentation, du reste en cours, pourra donc nous renseigner sur le comportement de l'Épicéa commun, soumis à de telles éclaircies, dans le nord-est de notre pays.

Nous ne reviendrons pas, enfin, sur les conditions d'utilisation de ces tables, ce sujet ayant été développé dans d'autres publications, notamment dans la Revue Forestière Française (Decourt N., 1964) ou dans le Bulletin de la Vulgarisation Forestière (Cf. no 71/2 - Février 1971).

Finalement, comme pour les premières tables publiées, on fera remarquer que ces tables sont provisoires et qu'elles seront améliorées et précisées dans les prochaines années, 


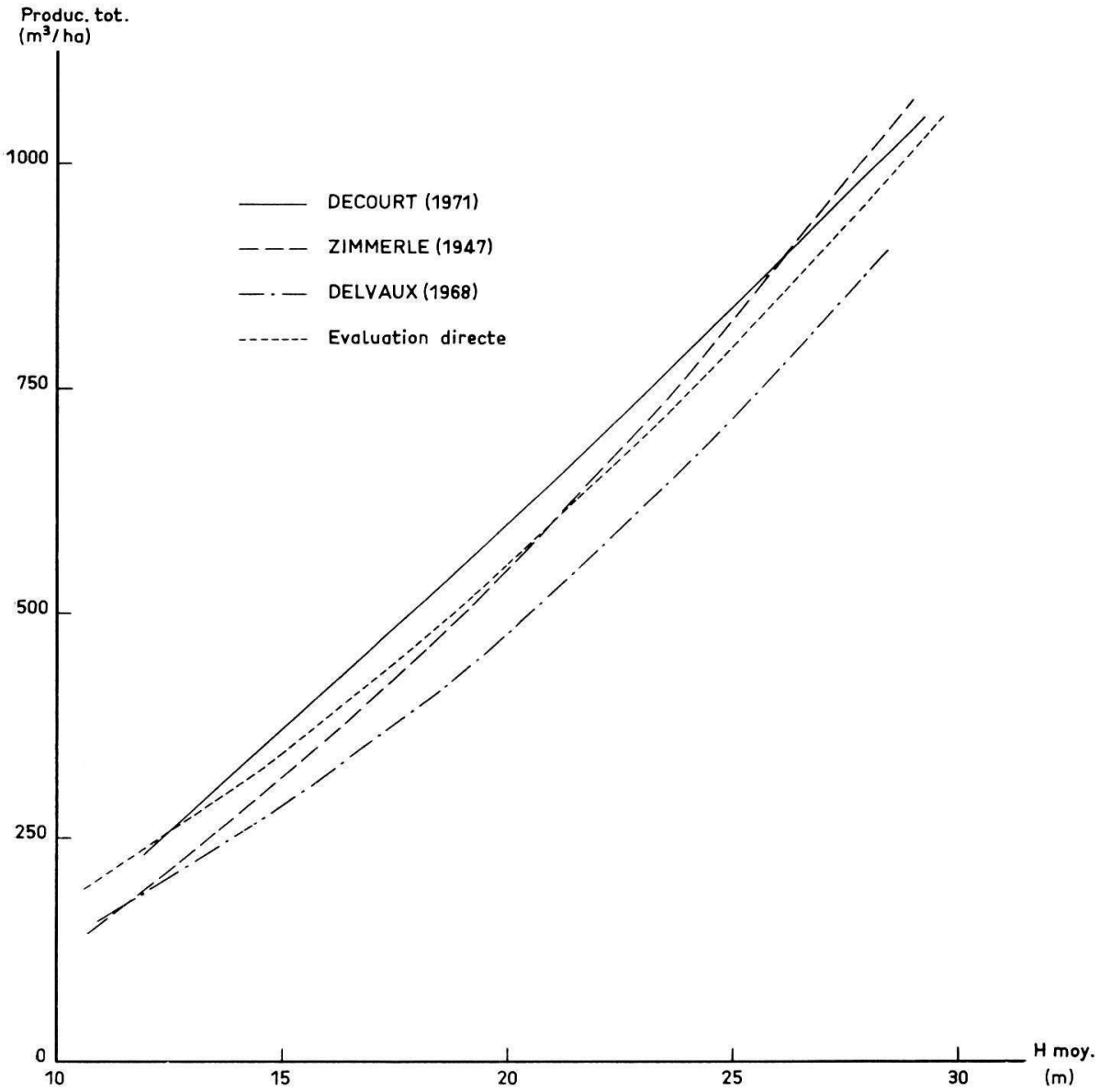

FIG. 4. - Production totale et croissance en hauteur en Ardennes Belges (Delvaux), en Württemberg (Zimmerle) et dans le N.-E. de la France

FIG. 4. - Total Yield and height growth in Belgian Ardennes (Delvaux), in Württemberg (Zimmerle) and in Northeast of France

TABLEAU 4

Limites de validité des équations utilisées

Validity limits of the equations utilized

\begin{tabular}{|c|c|c|c|c|}
\hline \multirow{2}{*}{$\begin{array}{c}\begin{array}{c}\text { Hauteur } \\
\text { moyenne }\end{array} \\
\mathrm{HG}\end{array}$} & \multicolumn{2}{|c|}{$\begin{array}{l}\text { Nombre de tiges } \\
\text { à l'hectare }\end{array}$} & \multicolumn{2}{|c|}{$\begin{array}{l}\text { Surfaces terrières } \\
\text { en } \mathrm{m}^{2} / \text { ha }\end{array}$} \\
\hline & $\mathrm{N}$ min. & $\mathrm{N} \max$ & $\mathrm{G} \min$. & $\mathrm{G} \max$ \\
\hline 12 & 2.200 & 5.000 & 16 & 40 \\
\hline 15 & 1.400 & 4.000 & 23 & 52 \\
\hline 18 & 900 & 2.900 & 27 & 60 \\
\hline 21 & 750 & 2.000 & 33 & 63 \\
\hline 24 & 500 & 1.600 & 37 & 66 \\
\hline 27 & 400 & 1.200 & 39 & 68 \\
\hline 30 & 370 & 900 & 40 & 69 \\
\hline 33 & 350 & 700 & 40 & 69 \\
\hline
\end{tabular}


à partir d'observations renouvelées dans le même réseau de placettes semi-permanentes.

En attendant, souhaitons que cette table, bien que provisoire, puisse rendre service pour la gestion des peuplements d'Épicéa commun du N.-E.

Reçu pour publication en octobre 1971.

\section{SUMMARY}

\section{PROVISIONAL YIELD-TABLES FOR NORWAY SPRUCE IN THE NORTHEAST OF FRANCE.} plots.

This yield-table concerns even-aged pure stands. It has been entirely computed from temporary

The method is based on the computation of relationships, by multiple regression method, using following variables : Age, mean-height, dominant height, number of stems per hectare, mean circumference, dominant circumference and stand volume. The equations enable the computation of fundamentals relations giving the growth of the height, the mean circumference and the volume.

From these relations and after the choice of a thinning regime, it is possible to calculate usual yield-tables.

\section{ZUSAMMENFASSUNG}

\section{VORLAÜFIGE FICHTEN-ERTRAGSTAFEL FÜR NORD-OST FRANKREICH}

Die vorliegend Ertragstafel wurde für Fichtenreinbestände ausschliesslich mit einmalig erhobenen Versuchsflächen aufgestellt.

Das angewandte Verfahren beruht auf der Berechnung von Mehrfachregressionsgleichungen unter Einbeziehung von Alter, Mittelhöhe, Oberhöhe, Stammzahl, mittlerer Umfang, Umfang des Kreisflächenmittelstammes der 100 stärksten Stämme pro ha und Vorratsmasse. Diese Gleichungen erlauben die Berechnung von Grundbeziehungen, die dem Höhenzuwachs, dem Umfangzuwachs und dem Massenzuwachs Rechnung tragen.

Ausgehend von diesen Grundbeziehungen und nach Wahl der Durchforstung werden die Tafeln in der bisher üblichen Art berechnet.

\section{RÉFÉRENCES BIBLIOGRAPHIQUES}

Dagnelle P., Nivelle J.-L., Rondeux J., Thill A., 1970. Production de l'Épicéa commun (Picea Abies Karst.) dans quelques stations de l'Ardenne centrale, à publier dans Bull. des Rech. Agr. de Gembloux. Vol. 5, 1970.

Decourt N., 1964. Les tables de production, leurs limites et leur utilité. R.F.F. août-septembre 1964, p. 640.

Decourt N., 1965. Le Pin sylvestre et le Pin Laricio de Corse en Sologne. Tables de production provisoires et méthodes utilisées pour les construire. Ann. Sci. Forestières, 22 (2), 259-318.

Decourt N., 1967. Le Douglas dans le Nord-Est du Massif Central. Tables de production provisoires. Ann. Sci. Forestières, 24 (1), 45-84.

Decourt N. et Lemoine B., 1969. Le Pin maritime dans le Sud-Ouest de la France. Tables de production provisoires. Ann. Sciences Forestières, 26 (1), 3-44.

Decourt N. et Le TACon F., 1970. L'épicéa commun sur les plateaux calcaires de l'Est de la France. Essai de prévision de la production à l'aide de déterminations pédologiques simples. Ann. Sci. Forestières, 27 (3), 255-286.

Decourt N., 1971. Comparaison des équations de régression. Application au cubage des peuplements d'Épicéa commun. Ann. Sci. Forestières (à l'impression).

Delvaux J., 1968. Picea Abies Karst. Production de 15 placettes permanentes en Belgique. Travaux Série Série B, no 34. St. Rech. des Eaux-et-Forêts. Grœnendaal-Hoeilaart, p. 49-59.

I.D.F., 1971. Bulletin de la Vulgarisation Forestière, n ${ }^{\circ} 71 / 2$.

KozaK A., 1970. A simple method to test parallelism and coincidence for curvilinear multiple linear and multiple curvilinear regressions. 3rd conf. of the advisory Group of Forest Statisticians Jouy-enJosas, France (sept. 7-11, 1970). 
ANNEXE 1

Définition des variables

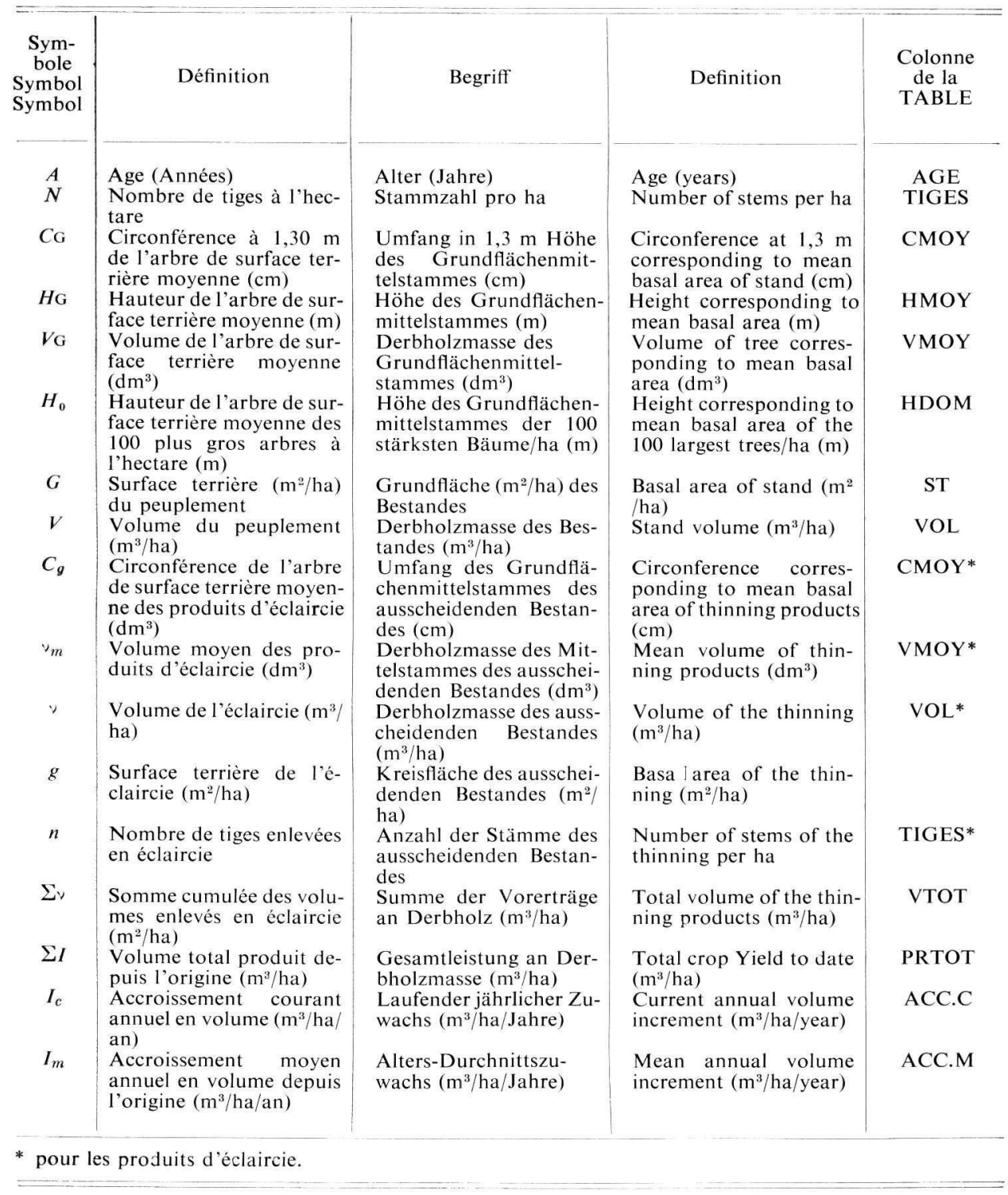


ANNEXE 2

Table de production-Épicéa dans le nord-est

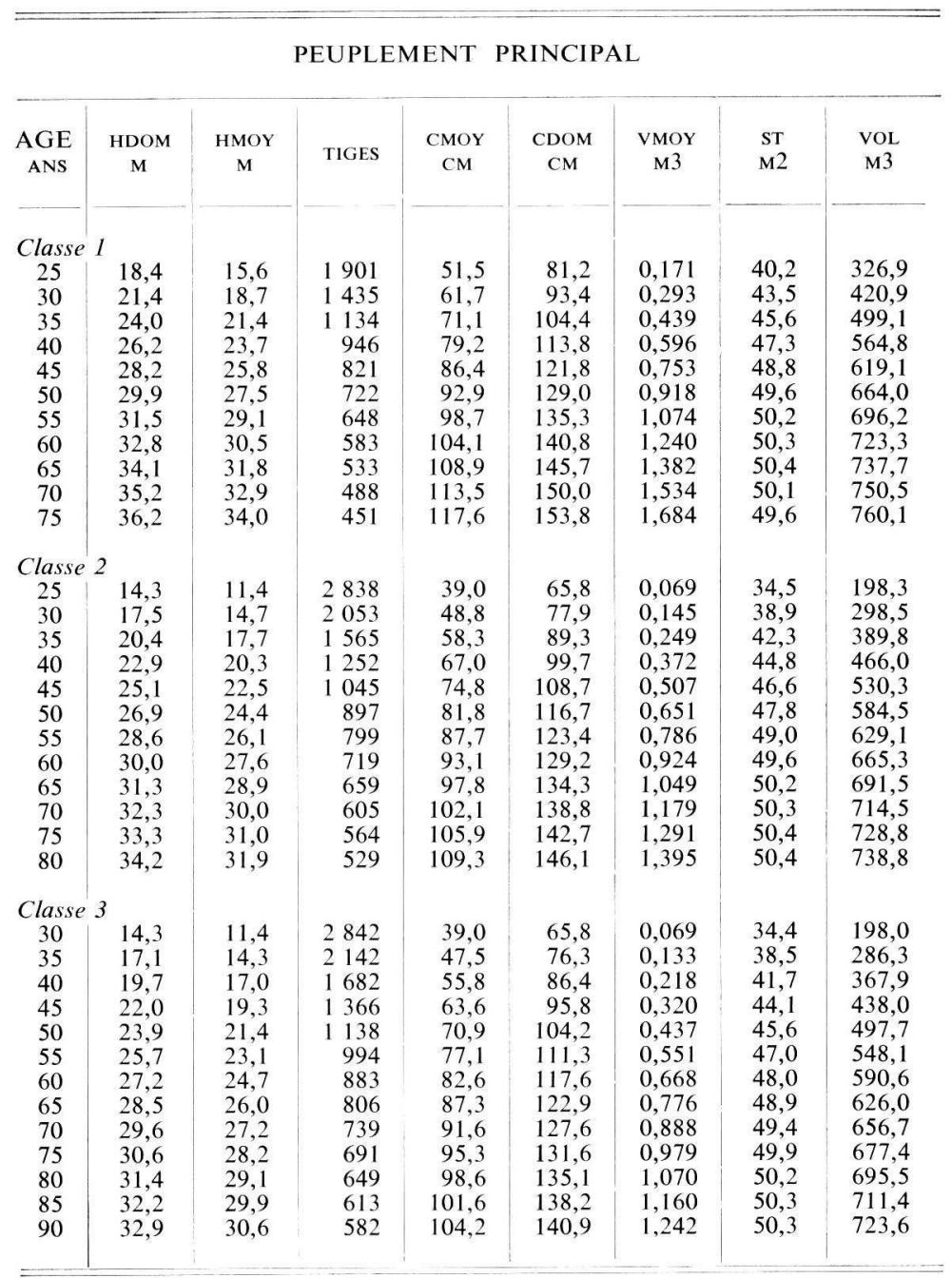




\begin{tabular}{|c|c|c|c|c|c|c|c|c|}
\hline \multicolumn{9}{|c|}{ ÉCLAIRCIES } \\
\hline TIGES & $\begin{array}{l}\text { CMOY } \\
\text { CM }\end{array}$ & $\begin{array}{c}\text { VMOY } \\
\text { M3 }\end{array}$ & $\begin{array}{l}\text { VOL } \\
\text { M3 }\end{array}$ & $\begin{array}{l}\text { VTOT } \\
\text { M3 }\end{array}$ & $\begin{array}{c}\text { ECL } \\
/ 100\end{array}$ & $\begin{array}{c}\text { PRTOT } \\
\text { M3 }\end{array}$ & $\begin{array}{l}\text { ACC.C } \\
\text { M3/AN }\end{array}$ & $\begin{array}{l}\text { ACC.M } \\
\text { M3/AN }\end{array}$ \\
\hline 739 & 27,2 & 0,095 & 70,5 & 70,5 & 17,7 & 397,5 & 32,2 & 15,9 \\
\hline 466 & 35,7 & 0,104 & 48,7 & 119,2 & 22,0 & 540,2 & 27,1 & 18,0 \\
\hline 301 & 43,9 & 0,169 & 50,9 & 170,2 & 25,4 & 669,3 & 24,3 & 19,1 \\
\hline 188 & 51,4 & 0,256 & 48,2 & 218,4 & 27,8 & 783,3 & 21,2 & 19,5 \\
\hline 125 & 57,8 & 0,356 & 44,5 & 263,0 & 29,8 & 882,1 & 18,4 & 19,6 \\
\hline 99 & 63,3 & 0,412 & 40,8 & 303,9 & 31,3 & 967,9 & 15,8 & 19,3 \\
\hline 74 & 68,1 & 0,543 & 40,2 & 344,1 & 33,0 & 1040,4 & 13,6 & 18,9 \\
\hline 65 & 72,3 & 0,560 & 36,4 & 380,5 & 34,4 & 1103,9 & 11,4 & 18,3 \\
\hline 50 & 76,2 & 0,727 & 36,3 & 416,9 & 36,1 & 1154,6 & 9,6 & 17,7 \\
\hline 45 & 79,6 & 0,727 & 32,7 & 449,6 & 37,4 & 1200,2 & 8,4 & 17,1 \\
\hline 37 & 82,8 & 0,804 & 29,7 & 479,4 & 38,6 & 1239,5 & 7,0 & 16,5 \\
\hline 1362 & 17,9 & 0,018 & 25,5 & 25,5 & 11,4 & 223,9 & 25,4 & 8,9 \\
\hline 785 & 24,7 & 0,040 & 31,8 & 57,3 & 16,1 & 355,9 & 26,5 & 11,8 \\
\hline 488 & 32,5 & 0,087 & 42,6 & 100,0 & 20,4 & 489,9 & 25,7 & 13,9 \\
\hline 313 & 40,4 & 0,152 & 47,8 & 147,8 & 24,0 & 613,9 & 23,3 & 15,3 \\
\hline 207 & 47,5 & 0,218 & 45,2 & 193,1 & 26,6 & 723,4 & 20,4 & 16,0 \\
\hline 148 & 53,7 & 0,276 & 40,9 & 234,0 & 28,5 & 818,6 & 17,7 & 16,3 \\
\hline 98 & 59,0 & 0,382 & 37,4 & 271,5 & 30,1 & 900,6 & 15,2 & 16,3 \\
\hline 80 & 63,5 & 0,425 & 34,0 & 305,5 & 31,4 & 970,8 & 12,9 & 16,1 \\
\hline 60 & 67,4 & 0,544 & 32,6 & 338,2 & 32,8 & 1029,7 & 11,0 & 15,8 \\
\hline 54 & 70,8 & 0,531 & 28,7 & 366,9 & 33,9 & 1081,4 & 9,3 & 15,4 \\
\hline 41 & 73,8 & 0,672 & 27,5 & 394,4 & 35,1 & 1123,3 & 7,7 & 14,9 \\
\hline 35 & 76,5 & 0,727 & 25,4 & 419,9 & 36,2 & 1158,8 & 6,7 & 14,4 \\
\hline 1152 & 17,9 & 0,022 & 25,4 & 25,4 & 11,3 & 223,5 & 22,6 & 7,4 \\
\hline 700 & 23,9 & 0,039 & 27,9 & 53,3 & 15,7 & 339,7 & 23,4 & 9,7 \\
\hline 460 & 30,7 & 0,079 & 36,4 & 89,7 & 19,6 & 457,7 & 22,8 & 11,4 \\
\hline 316 & 37,5 & 0,127 & 40,3 & 130,1 & 22,9 & 568,1 & 20,9 & 12,6 \\
\hline 228 & 43,8 & 0,171 & 39,1 & 169,2 & 25,3 & 667,0 & 18,6 & 13,3 \\
\hline 144 & 49,5 & 0,256 & 36,9 & 206,2 & 27,3 & 754,3 & 16,2 & 13,7 \\
\hline 111 & 54,4 & 0,296 & 32,9 & 239,1 & 28,8 & 829,8 & 14,0 & 13,8 \\
\hline 77 & 58,6 & 0,386 & 29,7 & 268,9 & 30,0 & 894,9 & 12,1 & 13,7 \\
\hline 67 & 62,2 & 0,386 & 25,8 & 294,8 & 30,9 & 951,5 & 10,3 & 13,5 \\
\hline 48 & 65,3 & 0,538 & 25,8 & 320,6 & 32,1 & 998,0 & 8,7 & 13,3 \\
\hline 42 & 68,0 & 0,538 & 22,6 & 343,2 & 33,0 & 1038,8 & 7,6 & 12,9 \\
\hline 36 & 70,3 & 0,551 & 19,8 & 363,1 & 33,7 & 1074,5 & 6,6 & 12,6 \\
\hline 31 & 72,4 & 0,585 & 18,1 & 381,2 & 34,5 & 1104,9 & 5,4 & 12,2 \\
\hline
\end{tabular}


Table de production-Épicéa dans le nord-est

PEUPLEMENT PRINCIPAL

\begin{tabular}{|c|c|c|c|c|c|c|c|c|}
\hline $\begin{array}{c}\mathrm{AGE} \\
\text { ANS }\end{array}$ & $\begin{array}{c}\text { HDOM } \\
\mathbf{M}\end{array}$ & $\begin{array}{c}\text { HMOY } \\
\text { M }\end{array}$ & TIGES & $\begin{array}{c}\text { CMOY } \\
\text { CM }\end{array}$ & $\begin{array}{c}\text { CDOM } \\
\mathbf{C M}\end{array}$ & $\begin{array}{c}\text { VMOY } \\
\text { M3 }\end{array}$ & $\begin{array}{l}\mathrm{ST} \\
\mathrm{M} 2\end{array}$ & $\begin{array}{l}\text { VOL } \\
\text { M3 }\end{array}$ \\
\hline \multicolumn{9}{|c|}{ Classe 4} \\
\hline 35 & 14,2 & 11,3 & 2870 & 38,7 & 65,5 & 0,068 & 34,3 & 195,7 \\
\hline 40 & 16,7 & 13,9 & 2244 & 46,1 & 74,5 & 0,121 & 37,9 & 272,2 \\
\hline 45 & 18,9 & 16,2 & 1811 & 53,2 & 83,3 & 0,189 & 40,9 & 343,7 \\
\hline 50 & 20,9 & 18,3 & 1491 & 60,2 & 91,6 & 0,273 & 43,0 & 407,2 \\
\hline 55 & 22,7 & 20,1 & 1272 & 66,4 & 99,0 & 0,362 & 44,7 & 461,1 \\
\hline 60 & 24,3 & 21,7 & 1111 & 72,0 & 105,5 & 0,456 & 45,9 & 507,4 \\
\hline 65 & 25,6 & 23,1 & 997 & 76,9 & 111,2 & 0,548 & 47,0 & 547,0 \\
\hline 70 & 26,8 & 24,3 & 904 & 81,4 & 116,1 & 0,642 & 47,7 & 581,0 \\
\hline 75 & 27,8 & 25,4 & 843 & 85,0 & 120,3 & 0,721 & 48,5 & 609,0 \\
\hline 80 & 28,7 & 26,3 & 790 & 88,3 & 124,0 & 0,801 & 49,1 & 633,4 \\
\hline 85 & 29,5 & 27,1 & 743 & 91,3 & 127,3 & 0,880 & 49,4 & 654,7 \\
\hline 90 & 30,2 & 27,8 & 709 & 93,9 & 130,1 & 0,943 & 49,8 & 669,5 \\
\hline \multicolumn{9}{|c|}{ Classe 5} \\
\hline 35 & 11,5 & 8,6 & 3834 & 31,5 & 56,3 & 0,031 & 30,3 & 121,6 \\
\hline 40 & 13,8 & 10,9 & 2994 & 37,7 & 64,1 & 0,061 & 33,9 & 185,5 \\
\hline 45 & 16,0 & 13,1 & 2404 & 43,9 & 71,9 & 0,104 & 37,0 & 250,4 \\
\hline 50 & 17,9 & 15,2 & 1979 & 50,1 & 79,5 & 0,157 & 39,6 & 312,3 \\
\hline 55 & 19,7 & 17,0 & 1677 & 55,9 & 86,5 & 0,219 & 41,7 & 368,8 \\
\hline 60 & 21,3 & 18,6 & 1447 & 61,3 & 93,0 & 0,288 & 43,4 & 417,9 \\
\hline 65 & 22,7 & 20,1 & 1275 & 66,3 & 98,9 & 0,360 & 44,6 & 460,3 \\
\hline 70 & 23,9 & 21,3 & 1140 & 70,8 & 104,1 & 0,436 & 45,5 & 497,2 \\
\hline 75 & 25,0 & 22,4 & 1050 & 74,6 & 108,5 & 0,503 & 46,5 & 528,7 \\
\hline 80 & 26,0 & 23,4 & 971 & 78,1 & 112,5 & 0,572 & 47,2 & 556,3 \\
\hline 85 & 26,8 & 24,3 & 907 & 81,2 & 116,0 & 0,639 & 47,6 & 580,1 \\
\hline 90 & 27,5 & 25,0 & 863 & 83,8 & 118,9 & 0,694 & 48,3 & 599,8 \\
\hline \multicolumn{9}{|c|}{ Classe 6} \\
\hline 40 & 11,2 & 8,2 & 4012 & 30,5 & 55,0 & 0,027 & 29,7 & 111,9 \\
\hline 45 & 13,1 & 10,2 & 3247 & 35,6 & 61,5 & 0,050 & 32,8 & 164,8 \\
\hline 50 & 14,9 & 12,1 & 2640 & 41,0 & 68,2 & 0,082 & 35,4 & 217,9 \\
\hline 55 & 16,7 & 13,8 & 2251 & 46,0 & 74,4 & 0,120 & 37,9 & 271,4 \\
\hline 60 & 18,2 & 15,4 & 1935 & 50,9 & 80,5 & 0,165 & 39,9 & 320,6 \\
\hline 65 & 19,6 & 16,9 & 1696 & 55,5 & 86,1 & 0,215 & 41,6 & 365,3 \\
\hline 70 & 20,9 & 18,2 & 1500 & 59,9 & 91,3 & 0,269 & 42,9 & 404,8 \\
\hline 75 & 22,0 & 19,3 & 1362 & 63,7 & 95,9 & 0,322 & 44,1 & 438,8 \\
\hline 80 & 23,0 & 20,4 & 1240 & 67,4 & 100,1 & 0,378 & 44,8 & 469,1 \\
\hline 85 & 23,9 & 21,3 & 1145 & 70,6 & 103,9 & 0,432 & 45,5 & 495,4 \\
\hline 90 & 24,6 & 22,1 & 1080 & 73,3 & 107,0 & 0,479 & 46,2 & 518,1 \\
\hline
\end{tabular}


ÉPICÉA： TABLES DE PRODUCTION

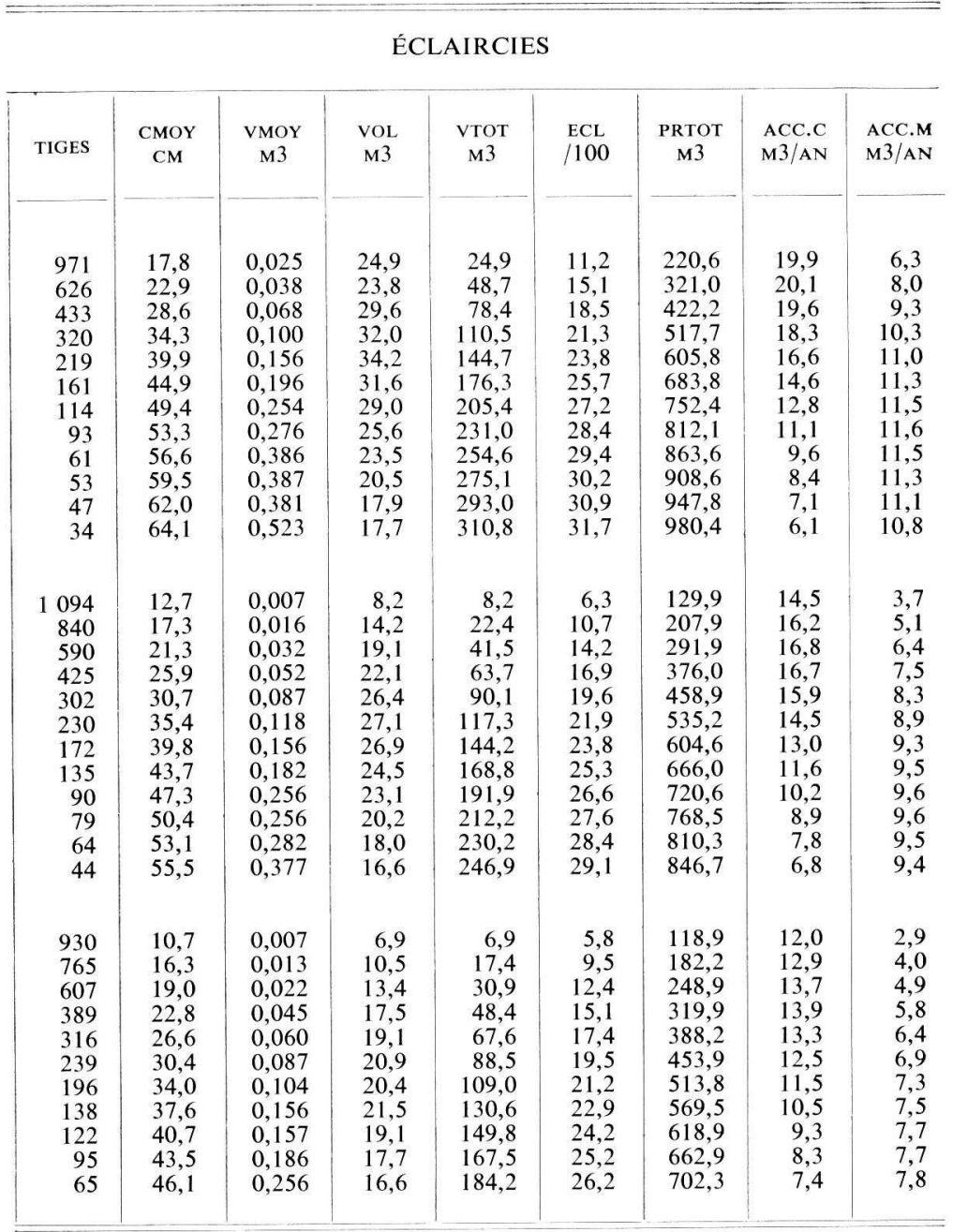

